\title{
Tedizolid, Faropenem, and Moxifloxacin Combination With Potential Activity Against Nonreplicating Mycobacterium tuberculosis
}

\author{
Shashikant Srivastava ${ }^{1,2,3 *}$, Kayle N. Cirrincione ${ }^{1}$, Devyani Deshpande ${ }^{1}$ and \\ Tawanda Gumbo ${ }^{1,4,5}$
}

${ }^{1}$ Center for Infectious Diseases Research and Experimental Therapeutics, Baylor Research Institute, Baylor University Medical Center, Dallas, TX, United States, ${ }^{2}$ Department of Immunology, UT Southwestern Medical Center, Dallas, TX, United States, ${ }^{3}$ Department of Pulmonary Immunology, University of Texas Health Science Center at Tyler, Tyler, TX, United States, ${ }^{4}$ Praedicare Laboratories and Quantitative Preclinical \& Clinical Sciences Department Praedicare Inc., Dallas, TX, United States, ${ }^{5}$ Department of Medicine, University of Cape Town, Observatory, Cape Town, South Africa

OPEN ACCESS

Edited by:

Lena Elisabeth Friberg, Uppsala University, Sweden

Reviewed by: Rob Van Wijk, Uppsala University, Sweden Amit P. Bhavsar, University of Alberta, Canada

*Correspondence: Shashikant Srivastava Shashi.kant@uthct.edu

Specialty section: This article was submitted to Translational Pharmacology, a section of the journal

Frontiers in Pharmacology

Received: 11 October 2020 Accepted: 26 November 2020 Published: 19 January 2021

Citation:

Srivastava S, Cirrincione KN, Deshpande D and Gumbo T (2021)

Tedizolid, Faropenem, and Moxifloxacin Combination With

Potential Activity Against Nonreplicating

Mycobacterium tuberculosis. Front. Pharmacol. 11:616294. doi: 10.3389/fphar.2020.616294
Background: Mycobacterium tuberculosis [Mtb] could be present in different metabolic population in the lung lesions, and nonreplicating persisters [NRP], associated with latent tuberculosis [TB], are the most difficult to kill.

Objective: Test the combination of tedizolid, moxifloxacin, and faropenem for activity against NRP using Mtb SS18b in the hollow fiber model [HFS-TB].

Methods: Tedizolid and moxifloxacin were tested as, first, two-drug combination against log-phase growth [LPG] and, second, slowly replicating bacilli [SRB] under acidic condition and with faropenem to create a three-drug combination regimen. Finally, standard regimen [isoniazid-rifampin-pyrazinamide] was used as comparator in the HFS-TB experiment with NRP Mtb. HFS-TB units were sampled for drug-concentration measurement as well as for estimation of bacterial burden using solid agar and mycobacterial growth indicator tube [MGIT] method. Linear regression was used to calculate the kill slopes with each treatment regimen and analysis of variance (ANOVA) to compare the regimen.

Results: Tedizolid at standard dose in combination with high-dose moxifloxacin killed 3.05 $\log _{10}$ CFU/ml LPG Mtb and $7.37 \log _{10}$ CFU/ml SRB in the bactericidal and sterilizing activity HFS-TB experiments, respectively. There was no statistical difference between tedizolid-moxifloxacin-faropenem combination and the standard regimen as both killed $7.35 \log _{10} \mathrm{CFU} / \mathrm{ml}$ NRP Mtb in 21 days. There was no emergence of resistance to any of the drugs studied in the three HFS-TB experiments.

Conclusion: The experimental regimen of tedizolid, moxifloxacin, and faropenem could effectively kill NRP population of Mtb, and given the efficacy against different metabolic population of Mtb could serve as a pan-TB regimen. Clinical studies are warranted to validate the in vitro findings.

Keywords: special population, tuberculosis, nonreplicating persisters, regimen, hollow fiber model 


\section{INTRODUCTION}

Explaining the basic mechanism of antituberculosis chemotherapy, Prof. Denis Mitchison in 1979 proposed the special population hypothesis, where each drug in the combination therapy had efficacy against a specific metabolic population of Mycobacterium tuberculosis [Mtb] (Mitchison, 1979). Isoniazid was suggested to be most active against the actively replicating bacilli, rifampin, and pyrazinamide having sterilizing activity against bacteria growing under acidic condition, and lack of drugs to have activity against dormant bacilli [nonreplicating persisters, NRP] (Mitchison, 1979). However, Mandal et al. (2019) recently published a review summarizing the problem of persisters in tuberculosis and the difficulties they posed to the tuberculosis drug discovery as well as potential role of pyrazinamide against this metabolic population. It is estimated that nearly one-third of the world's population is latently infected. $M t b$ can enter a dormant or latent state that is characterized by limited growth and metabolism and, consistent with Prof. Mitchison's hypothesis, this metabolic state displays phenotypic resistance to many of the commonly used drugs for treating tuberculosis [TB] (Chan and Flynn, 2004; Boshoff et al., 2005). This allows $M t b$ to persist indefinitely in the human host.

In the recent years, progress has been made to develop new models to study the efficacy of the drugs against NRP $M t b$. One such tool is streptomycin-starved Mtb 18b [SS18b] strain that grows only when streptomycin is present in the growth medium (Zhang et al., 2012). Using the SS18b strain, Zhang et al. tested in vitro killing efficacy of 22 drugs against this special $M t b$ metabolic population and found that some of the first-line antituberculosis drugs [rifamycins and pyrazinamide] as well as new/repurposed drugs [e.g., tedizolid] have efficacy against SS18b, hence against latent TB (Zhang et al., 2012). Another recent review article summarized the killing efficacy of the abovementioned drugs as well as that of moxifloxacin, isoniazid, and carbapenems alone or in combination of a beta-lactamase inhibitor (Iacobino et al., 2017). There was a dose-dependent killing of NRP with these drugs.

In order to access the extent of kill of NRP with three of the above-mentioned antibiotics, namely, tedizolid, moxifloxacin, and faropenem, that we found to be effective against log-phase growth [LPG] and semidormant bacilli [SRB] growing under acidic condition as well as against intracellular M. tuberculosis, we performed pharmacokinetics/pharmacodynamics studies using the hollow fiber model system of tuberculosis [HFS-TB] (Gumbo et al., 2009; Srivastava and Gumbo, 2011; Deshpande et al., 2016a; Swaminathan et al., 2016; Srivastava et al., 2018). HFS-TB is a preclinical drug development tool that can mimic human-like concentration-profile of the drugs and different half-life of drugs in the same systems while testing combination therapy regimens, approved by the European Medicines Agency, editorially endorsed by the United States FDA and have a predictive accuracy of over 94\% (Chilukuri et al., 2015; EuropeanMedicines-Agencies, 2015; Gumbo et al., 2015). Regarding the mechanism of action of the drugs we used in the current study, tedizolid works via inhibition of bacterial protein synthesis by binding to $23 \mathrm{~S}$ ribosomal RNA (rRNA) of the 50S subunit of the ribosome (Zhanel et al., 2015), moxifloxacin's bactericidal action occurs via preventing replication, transcription, and repair of bacterial DNA by binding to the topoisomerase enzymes II (DNA gyrase) (Ginsburg et al., 2003), and faropenem, that has strong affinity for the high molecular penicillin binding proteins of the bacteria cell wall, inhibits transpeptidation that results in inhibition of cell wall biosynthesis of the bacteria (Gettig et al., 2008). Therefore, combining these three drugs with different mechanism of action as well as potential synergy could create an effective treatment regimen with minimal likelihood of developing cross-resistance.

\section{MATERIALS AND METHODS}

\section{Bacteria, Drug, and Supplies}

All experiments were performed in a biosafety level 3 laboratory following the protocols approved by the Baylor Research Institute Biosafety Committee. We used Mtb H37Rv (ATCC27294) in the bactericidal activity and sterilizing activity experiments at acidic $\mathrm{pH}$ of 5.8 with detailed method as published elsewhere (Gumbo et al., 2009). Mtb SS18b strain (streptomycin auxotroph) was used in the NRP HFS-TB study. Streptomycin at $50 \mathrm{mg} / \mathrm{L}$ concentration was added to the growth medium of $M t b$ SS18b strain, a prerequisite growth condition as described earlier (Zhang et al., 2012). Tedizolid (the active moiety of the prodrug tedizolid phosphate) and faropenem were purchased from BOC Sciences (Shirley, NY), moxifloxacin from Baylor Medical Center Pharmacy, and isoniazid, rifampin, and pyrazinamide were purchased from Sigma Aldrich (St. Louis, MO). Hollow fiber cartridges were purchased from FiberCell Systems Inc. (Frederick, MD). Becton Dickinson (Franklin Lakes, NJ) was used to purchase the mycobacterial growth tube indicator system (BACTEC $^{\text {TM }}$ MGIT $^{\mathrm{TM}}$ 960) and related supplies.

\section{Tedizolid, Moxifloxacin, and Faropenem as Two- and Three-Drug Combination Compared to the Standard Therapy}

Before performing the HFS-TB experiments, MIC of the moxifloxacin, tedizolid, and faropenem [concentration range 0 , $0.06,0.12,0.25,0.5,1,2,4$, and $8 \mathrm{mg} / \mathrm{L}]$ was determined using the broth microdilution method (CLSI, 2018). We performed three different sets of hollow fiber experiments with three different $M$. tuberculosis metabolic populations, namely, actively replicating log-phase growth [LPG] bacilli at normal $\mathrm{pH}$, slowly replicating bacteria [SRB] under acidic $\mathrm{pH}$ of 5.8, and nonreplicating persisters [NRP]. The details of the HFS-TB and methods to transform the LPG to SRB cultures have been published previously and were used without any modifications (Gumbo et al., 2009; Srivastava and Gumbo, 2011).

In the first HFS-TB study, two-drug combination of tedizolid and moxifloxacin was tested for bactericidal activity using $20 \mathrm{ml}$ of LPG cultures inoculated into the peripheral compartment of each HFS-TB and treated with combination of tedizolid at standard dose of $200 \mathrm{mg}$ and moxifloxacin high dose of 
$800 \mathrm{mg}$ daily for 21 days. We mimicked a 12-h half-life for both tedizolid and moxifloxacin (Deshpande et al., 2016a; Srivastava et al., 2018). The central compartments of the HFS-TB receiving drug treatment were sampled at $0,1,4,6,10,18,21$, and $23.5 \mathrm{~h}$ after starting the drug treatment to measure the drug concentration for validation of the intended concentrationtime profile of the drugs. The peripheral compartments were sampled on days $0,7,10,14$, and 21 . The samples were washed twice to remove carry-over drug, serially 10 -fold diluted, and $200 \mu \mathrm{L}$ of the processed sample was inoculated on Middlebrook 7H10 agar supplemented with $10 \%$ oleic acid-albumin-dextrosecatalase (OADC) (herein termed "agar") to enumerate the total bacterial burden. The plates were incubated at $37^{\circ} \mathrm{C}$ in incubators with $5 \% \mathrm{CO}_{2}$ for 21 days after which the colony forming units (CFU) were recorded. One portion of the sample was also inoculated into the MGIT tubes and time to positive (TTP) was recorded to confirm the growth of $M t b$. We set the incubation period of the MGIT tubes or the "time in protocol" to 56 days in order to capture the relapse of failure of the regimens (Deshpande et al., 2016b). To capture the drug resistant subpopulation, the same samples were cultured on agar supplemented with $3 \mathrm{X}$ MIC of the respective drugs. The cultures were incubated for 28 days before the CFUs were recorded. Nontreated control systems received no drug treatment.

In the second HFS-TB study, the two-drug combination of tedizolid [standard dose] plus high dose moxifloxacin [800 mg/ daily] was tested for sterilizing activity over 42 days, two replicate HFS-TB units per treatment regimen. Twenty $\mathrm{mL}$ of the SRB culture was inoculated into the peripheral compartment of each of the HFS-TB units. Sampling of the central compartment was performed as described above to validate the concentration-time profile of tedizolid and moxifloxacin. The peripheral compartments were sampled on days $0,3,7,14,21,28,35$, and 42 and samples were processed to determine the total and drug resistant bacterial burden as described above, as well as TTP by inoculating the MGIT tubes.

In the third set of HFS-TB experiment, the M. tuberculosis SS18b strain was transformed to the NRP state by culturing in media without streptomycin supplementation (Zhang et al., 2012) and treated with either three-drug experimental combination of tedizolid, moxifloxacin, and faropenem or the standard regimen consisting of isoniazid plus rifampin plus pyrazinamide. There were three replicate HFS-TB units per treatment regimen. The half-lives of the drugs mimicked in the HFS-TB were $12 \mathrm{~h}$ for tedizolid, moxifloxacin, and pyrazinamide; $3 \mathrm{~h}$ for isoniazid and rifampin; and $1 \mathrm{~h}$ for faropenem (Srivastava et al., 2011a; Zhang et al., 2012; Deshpande et al., 2016a). Since the experiments were carried out in acidic environment using acidified media to a $\mathrm{pH}$ of 5.8, this set of experiments also served as a validation of the sterilizing activity in addition to determination of the killing efficacy of this combination against the NRP subpopulation of M. tuberculosis. Twenty $\mathrm{mL}$ of the NRP cultures were inoculated into the peripheral compartment of each of the HFS-TB units. The treatment regimens were as follows: standard dose of tedizolid once daily plus $800 \mathrm{mg}$ of moxifloxacin daily plus faropenem twice daily to achieve $66 \%$ time-above-MIC (\% $\mathrm{T}_{\mathrm{MIC}}$ ) (Deshpande et al., 2016a); three-drug combination of isoniazid $300 \mathrm{mg} /$ day, rifampin $600 \mathrm{mg} /$ day, and pyrazinamide $1.5 \mathrm{~g} /$ day; and nontreated controls. All HFS-TB except the nontreated controls were sampled for drug concentration measurements and enumeration of the bacterial burden and TTP as described above. Sampling of the central compartment to validate the concentration-time profile of the drugs and of the peripheral compartment on days $0,3,7,14,21,28,35,42,49$, and 56 was performed to determine the total and drug resistant bacterial burden as described above. The MGIT tubes were also inoculated to record the TTP as indication of $M t b$ growth.

\section{Pharmacokinetics/Pharmacodynamics Analysis}

We used previously published methods, without any change, to measure the drug concentrations in each of the HFS-TB samples (Srivastava et al., 2011a; Srivastava et al., 2011b; Deshpande et al., 2016a; Srivastava et al., 2018). ADAPT (D'Argenio and Schumitzky, 1997) was used for pharmacokinetic analysis with the modeling steps as described in the past, including the onecompartment model with first-order input and elimination (Gumbo et al., 2009; Srivastava et al., 2011a). We used two measures of bacterial burden: total $M t b \log _{10} \mathrm{CFU} / \mathrm{ml}$ and TTP in days. The linear regression model [for the CFU/ml readouts] was used to calculate the kill slopes of the combination regimens and one-way analysis of variance (ANOVA) was used to compare the regimens where the CFU readouts and therapeutic regimens were used as the dependent and independent variable, respectively. All the statistical analysis was performed using GraphPad Prism v8 (La Jolla, CA, United States).

\section{RESULTS}

The drug concentration-profiles achieved in the HFS-TB in three different experiments are shown in Supplementary Figures 1A-E, and the regression between the observed vs. model predicted concentration is shown in Supplementary Figure 1F where an $r^{2}=0.93$ shows good model fit and minimal bias.

The MIC of tedizolid and moxifloxacin against Mtb H37Rv was $0.25 \mathrm{mg} / \mathrm{l}$, whereas the MIC of tedizolid, moxifloxacin, and faropenem against SS18b strain were $0.25,0.5$, and $4 \mathrm{mg} / \mathrm{l}$, respectively. The changes in the bacterial burden over time, measured using TTP and $\mathrm{CFU} / \mathrm{ml}$, are shown in Figure 1. Figure 1A show the CFU/ml readouts in the HFS-TB where no bacterial growth was recorded on study day 10 . The results of the MGIT-derived [TTP] readouts for the bactericidal activity of HFS-TB using the LPG cultures are shown in Figure 1B. The higher the bacterial burden in the HFS-TB, lower the TTP. After 14 days of treatment with tedizolid plus high-dose moxifloxacin combination, the MGIT show no growth units after 56 days of incubation, indicating total microbial kill in the drug-treated HFS-TB units. The day-21 TTP readouts could not be 


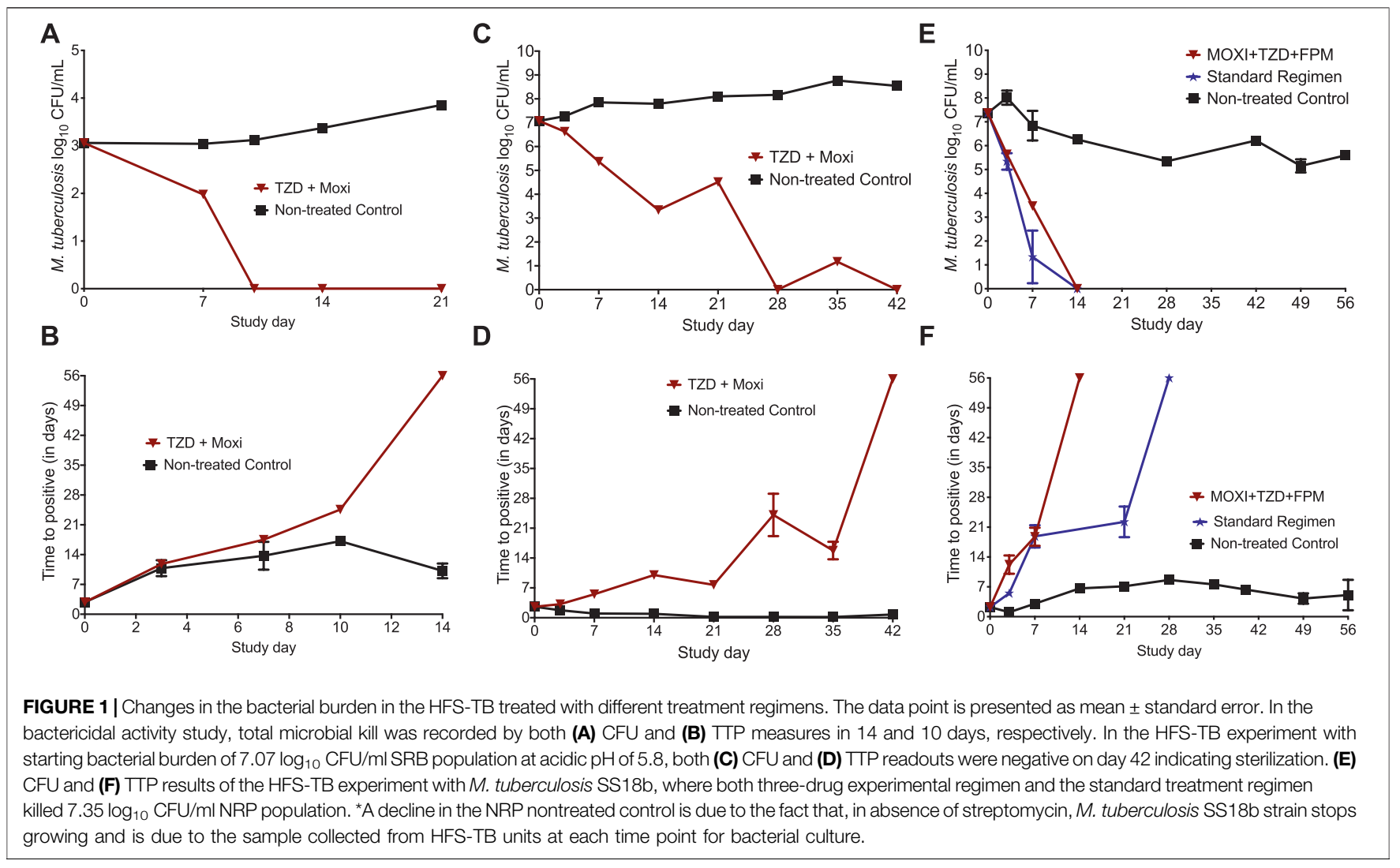

TABLE 1 | Growth rate of $M$. tuberculosis in nontreated HFS-TB and kill rate with experimental and standard regimen.

\begin{tabular}{ccccc} 
& TZD-MOXI & $\begin{array}{c}\text { TZD- } \\
\text { MOXI-FPM }\end{array}$ & $\begin{array}{c}\text { Standard } \\
\text { therapy }\end{array}$ & $\begin{array}{c}\text { Nontreated } \\
\text { control }\end{array}$ \\
\hline LPG & $-0.28 \pm 0.12$ & Not tested & Not tested & $0.04 \pm 0.011$ \\
SRB & $-0.17 \pm 0.027$ & Not tested & Not tested & $0.035 \pm 0.01$ \\
NRP & Not tested & $-0.53 \pm 0.09$ & $-0.59 \pm 0.08$ & $-0.039 \pm 0.01$
\end{tabular}

TZD, tedizolid; MOXI, moxifloxacin; FPM, faropenem; LPG, log-phase growth; SRB, slowly replicating bacilli at acidic $\mathrm{pH}$ of 5.8; NRP, nonreplicating persisters. The growth and kill rates are given as $\log _{10} \mathrm{CFU} / \mathrm{mL} /$ day.

recorded due to a technical error; however, given that prior time point showed no growth units, it is assumed that there were no viable bacteria left in the systems.

The results of the sterilizing activity study, with $M t b$ cultures growing under acidic condition, are shown in Figures 1C,D. The reduction in total bacterial counts, as $\log _{10} \mathrm{CFU} / \mathrm{ml}$, is shown in Figure 1C. No bacterial colony was recorded on day 28 , followed by a transient regrowth on day 35 . The TTP readouts (Figure 1C) showed that sterilization was achieved after 42 days of treatment with tedizolid plus high-dose moxifloxacin. Since MGIT is more sensitive compared to the solid agar-based culture method, we considered that sterilization of the HFS-TB units inoculated with the SRB $M$. tuberculosis was achieved after 42 days of treatment with tedizolid-moxifloxacin dual combination therapy.
In Figures 1E,F, we show the results of the three-drug combination used to treat the NRP Mtb SS18b. By the CFU measure (Figure 1E), both three-drug regimens killed the total NRP population in 14 days. As shown in Figure 1E, when TTP was used as a pharmacodynamics measure, the three-drug combination of tedizolid-moxifloxacin-faropenem showed negative culture after 14 days of therapy compared to the 21 days with the standard treatment regimen of isoniazidrifampin-pyrazinamide. The kill rate with two- and three-drug regimens, calculated using the linear regression, is shown in Table 1. A negative growth rate in the NRP nontreated control (Table 1) is due to the fact that, in absence of streptomycin, $M$. tuberculosis SS18b strain stopped growing and a decline in total bacterial burden is due to the sample collected from HFS-TB units at each time point. Next, one-way ANOVA analysis was performed to compare the treatment regimens using the $\mathrm{CFU}$ readouts as dependent variable. The difference in the microbial kill between the standard and the experimental regimen was not significantly different $[p=0.671]$. There were no drug resistance colonies recorded in the three HFS-TB experiments for tedizolid, moxifloxacin, and faropenem on agar supplemented with $3 \mathrm{X}$ MIC concentration.

\section{DISCUSSION}

Since people with latent tuberculosis could develop active TB at some point of life when the immune system gets compromised, 
this population essentially serve as a reservoir for TB. Therefore, finding drugs and combination that can effectively kill NRP $M t b$ is crucial (Barry et al., 2009). There are several major findings in the present study. First, using the human-like pharmacokinetics of the drugs, we show that tedizolid and moxifloxacin combination can effectively kill different metabolic populations of $M t b$, in essence rejecting the special population hypothesis (Mitchison, 1979) that drugs work on selected Mtb metabolic states. Second, the three-drug combination of tedizolid-moxifloxacin-faropenem is effective against the NRP $M t b$, as confirmed by killing of 7.37 $\log _{10} \mathrm{CFU} / \mathrm{ml}$ after 21 days of treatment [using the TTP readouts]. Thus, this three-drug combination could be used in the clinics. Since, tedizolid, moxifloxacin, and faropenem are active against both drug susceptible and drug resistant $M t b$, this combination regimen has a potential to be pan-TB regimen. However, studies on drug resistant strains remain to be done, as well as clinical validation.

The third finding is the NRP killing efficacy of the isoniazidrifampin-pyrazinamide. Previously it was shown that the extent of NRP kill with rifampin varies in a dose-dependent manner (Zhang et al., 2012; Iacobino et al., 2017), isoniazid has minimal activity (Srivastava et al., 2011b; Swaminathan et al., 2016), and pyrazinamide barely had any effect against $M t b$ SS18b or NRP state (Zhang et al., 2012). We found that the standard therapy killed $7.35 \log _{10} \mathrm{CFU} / \mathrm{ml}$ NRP $M t b$ in 21 days, where the kill was likely driven by rifampin. This finding, while goes against the conventional wisdom (Mitchison, 1979) that none of the drugs in the standard regime kill NRP or dormant $M t b$, likely explains why the currently recommended standard therapy successfully cures a large proportion of patients.

Our study has some limitation. First, the starting bacterial burden in the bactericidal activity HFS-TB experiment was lower than intended. Thus, the faster kill rate of LPG with tedizolid and moxifloxacin combination may not truly represent the scenario with higher bacterial load in the lung lesions. Second, in the nontreated HFS-TB, the bacteria in the log-phase growth experiment grew only $0.796 \log _{10} \mathrm{CFU} / \mathrm{ml}$ in 21 days. We are unsure how this slow growth rate might have affected the outcome of the bactericidal activity of the drug combination. Third, we did not test emergence of drug resistance to isoniazid, rifampin, and pyrazinamide. However, since the total bacterial population was killed by the three-drug standard combination

\section{REFERENCES}

Barry, C. E., 3rd, Boshoff, H. I., Dartois, V., Dick, T., Ehrt, S., Flynn, J., et al. (2009). The spectrum of latent tuberculosis: rethinking the biology and intervention strategies. Nat. Rev. Microbiol. 7, 845-855. doi:10.1038/nrmicro2236

Boshoff, H. I., and Barry, C. E., 3rd (2005). Tuberculosis - metabolism and respiration in the absence of growth. Nat. Rev. Microbiol. 3, 70-80. doi:10.1038/nrmicro1065

Chan, J., and Flynn, J. (2004). The immunological aspects of latency in tuberculosis. Clin. Immunol. 110, 2-12. doi:10.1016/s1521-6616(03)00210-9

Chilukuri, D., McMaster, O., Bergman, K., Colangelo, P., Snow, K., and Toerner, J. G. (2015). The hollow fiber system model in the nonclinical evaluation of antituberculosis drug regimens. Clin. Infect. Dis. 61 (Suppl. 1), S32-S33. doi:10. 1093/cid/civ460 regimen, it is unlikely that there would be any acquired drug resistance to these drugs.

To conclude, the experimental combination regimen of tedizolid, moxifloxacin, and faropenem could effectively kill NRP population of $M t b$, and currently recommended standard regimen has equally good efficacy against NRP in the HFS-TB model. The clinical validation of these findings remains to be determined.

\section{DATA AVAILABILITY STATEMENT}

The raw data supporting the conclusions of this article will be made available by the authors, without undue reservation following institutional policies.

\section{AUTHOR CONTRIBUTIONS}

SS and TG designed the study; SS, KC, and DD performed the experiments; SS wrote the first draft of the manuscript. All authors read and approved the final version of the manuscript.

\section{FUNDING}

This work was supported by the Eunice Kennedy Shriver National Institute of Child Health and Human Development (NICHD; 1R01HD099756-02) to SS.

\section{ACKNOWLEDGMENTS}

We are thankful to S. Cole, Ecole Polytechnique Fédérale de Lausanne, Switzerland, for providing SS18b strain.

\section{SUPPLEMENTARY MATERIAL}

The Supplementary Material for this article can be found online at: https://www.frontiersin.org/articles/10.3389/fphar.2020.616294/ full\#supplementary-material.

CLSI (2018). "Susceptibility testing of mycobacteria, nocardia spp., and other aerobic actinomycetes," in CLSI standard M24. 3rd Edn (Wayne, PA: Clinical and Laboratory Standards Institute).

D'argenio, D. Z., and Schumitzky, A. (1997). ADAPT II. A program for simulation, identification, and optimal experimental design. User manual. Biomedical Simulations Resource. Los Angeles, California, USA: University of Southern California.

Deshpande, D., Srivastava, S., Nuermberger, E., Pasipanodya, J. G., Swaminathan, S., and Gumbo, T. (2016). A Faropenem, linezolid, and moxifloxacin regimen for both drug-susceptible and multidrug-resistant tuberculosis in children: FLAME path on the milky way. Clin. Infect. Dis. 63, S95-S101. doi:10.1093/ $\mathrm{cid} / \mathrm{ciw} 474$

Deshpande, D., Srivastava, S., Pasipanodya, J. G., Bush, S. J., Nuermberger, E., Swaminathan, S., et al. (2016). Linezolid for infants and toddlers with 
disseminated tuberculosis: first steps. Clin. Infect. Dis. 63, S80-S87. doi:10.1093/ cid/ciw482

European-Medicines-Agencies (2015). Qualification opinion on in-vitro hollowfibre-system model of tuberculosis (HFS-TB) [Online]. Available: http://www. ema.europa.eu/ema/index.jsp?curl=pages/includes/document/document_ detail.jsp? webContentId=WC500181899\&mid=WC0b01ac058009a3dc (Accessed January 31, 2017).

Gettig, J. P., Crank, C. W., and Philbrick, A. H. (2008). Faropenem medoxomil. Ann. Pharmacother. 42, 80-90. doi:10.1345/aph.1G232

Ginsburg, A. S., Grosset, J. H., and Bishai, W. R. (2003). Fluoroquinolones, tuberculosis, and resistance. Lancet Infect. Dis. 3, 432-442. doi:10.1016/ s1473-3099(03)00671-6

Gumbo, T., Dona, C. S., Meek, C., and Leff, R. (2009). Pharmacokineticspharmacodynamics of pyrazinamide in a novel in vitro model of tuberculosis for sterilizing effect: a paradigm for faster assessment of new antituberculosis drugs. Antimicrob. Agents Chemother. 53, 3197-3204. doi:10.1128/AAC.01681-08

Gumbo, T., Pasipanodya, J. G., Romero, K., Hanna, D., and Nuermberger, E. (2015). Forecasting accuracy of the hollow fiber model of tuberculosis for clinical therapeutic outcomes. Clin. Infect. Dis. 61 (Suppl. 1), S25-S31. doi:10. 1093/cid/civ427

Iacobino, A., Piccaro, G., Giannoni, F., Mustazzolu, A., and Fattorini, L. (2017). Fighting tuberculosis by drugs targeting nonreplicating Mycobacterium tuberculosis bacilli. Int. J. Mycobacteriol. 6, 213-221. doi:10.4103/ijmy.jimy_85_17

Mandal, S., Njikan, S., Kumar, A., Early, J. V., and Parish, T. (2019). The relevance of persisters in tuberculosis drug discovery. Microbiology (Read.). 165, 492-499. doi:10.1099/mic.0.000760

Mitchison, D. A. (1979). Basic mechanisms of chemotherapy. Chest. 76, 771-781. doi:10.1378/chest.76.6_supplement.771

Srivastava, S., and Gumbo, T. (2011). In vitro and in vivo modeling of tuberculosis drugs and its impact on optimization of doses and regimens. Curr. Pharmaceut. Des. 17, 2881-2888. doi:10.2174/138161211797470192

Srivastava, S., Pasipanodya, J. G., Meek, C., Leff, R., and Gumbo, T. (2011). Multidrug-resistant tuberculosis not due to noncompliance but to betweenpatient pharmacokinetic variability. J. Infect. Dis. 204, 1951-1959. doi:10.1093/ infdis/jir658
Srivastava, S., Sherman, C., Meek, C., Leff, R., and Gumbo, T. (2011). Pharmacokinetic mismatch does not lead to emergence of isoniazid- or rifampin-resistant Mycobacterium tuberculosis but to better antimicrobial effect: a new paradigm for antituberculosis drug scheduling. Antimicrob. Agents Chemother. 55, 5085-5089. doi:10.1128/AAC.00269-11

Srivastava, S., Deshpande, D., Nuermberger, E., Lee, P. S., Cirrincione, K., Dheda, K., et al. (2018). The sterilizing effect of intermittent tedizolid for pulmonary tuberculosis. Clin. Infect. Dis. 67, S336-S341. doi:10.1093/cid/ciy626

Swaminathan, S., Pasipanodya, J. G., Ramachandran, G., Hemanth Kumar, A. K., Srivastava, S., Deshpande, D., et al. (2016). Drug concentration thresholds predictive of therapy failure and death in children with tuberculosis: bread crumb trails in random forests. Clin. Infect. Dis. 63, S63-S74. doi:10.1093/cid/ciw471

Zhanel, G. G., Love, R., Adam, H., Golden, A., Zelenitsky, S., Schweizer, F., et al. (2015). Tedizolid: a novel oxazolidinone with potent activity against multidrugresistant gram-positive pathogens. Drugs 75, 253-270. doi:10.1007/s40265-0150352-7

Zhang, M., Sala, C., Hartkoorn, R. C., Dhar, N., Mendoza-Losana, A., and Cole, S. T. (2012). Streptomycin-starved Mycobacterium tuberculosis 18b, a drug discovery tool for latent tuberculosis. Antimicrob. Agents Chemother. 56, 5782-5789. doi:10.1128/AAC.01125-12

Conflict of Interest: TG founded Praedicare Inc. and is on the Governing Board of Kiara Health.

The remaining authors declare that the research was conducted in the absence of any commercial or financial relationships that could be construed as a potential conflict of interest.

Copyright (C 2021 Srivastava, Cirrincione, Deshpande and Gumbo. This is an openaccess article distributed under the terms of the Creative Commons Attribution License (CC BY). The use, distribution or reproduction in other forums is permitted, provided the original author(s) and the copyright owner(s) are credited and that the original publication in this journal is cited, in accordance with accepted academic practice. No use, distribution or reproduction is permitted which does not comply with these terms. 